\title{
Generación de un modelo de calidad para la evaluación de Objetos de Aprendizaje utilizando notación $i^{\star}$ e ISO/IEC 25010
}

\section{(Generation of a Quality Model for Learning Objects Using the iStar Notation and the ISO/IEC 25010 Standard)}

\begin{abstract}
Vanessa Solis Cabrera ${ }^{1}$, Juan Pablo Carvallo², Jorge Maldonado-Mahauad ${ }^{1}$
Resumen:

Este trabajo describe el proceso para la creación de un modelo de calidad que evalúe Objetos de Aprendizaje (OA) en aspectos tecnológicos y didácticos utilizando la notación $i^{*}$. Para cumplir con este objetivo se revisaron modelos de calidad en ambos aspectos y conjuntamente con los conceptos de OA y calidad se han determinado las características que debe cumplir un OA. Para entender el contexto de un OA se definió un modelo de Dependencias Estratégicas (SD) utilizando la notación $i^{*}$. Con el modelo SD creado se establece la trazabilidad entre sus criterios y los elementos de calidad incluidos en el estándar ISO/IEC 25010. El modelo de calidad resultante fue enriquecido con las características y los atributos identificados en la literatura utilizando el método IQMC. A cada propiedad de calidad incluida se le asignaron métricas apropiadas para su evaluación. Como resultado se obtuvo un modelo genérico para la evaluación de distintos tipos de OA
\end{abstract}

Palabras clave: modelo calidad; notación $i^{*}$; objeto de aprendizaje; IQMC; ISO/IEC 25010.

\begin{abstract}
:
This study describes the process for the creation of a quality model that evaluates Learning Objects in terms of technology and didactics by using $i^{*}$ notation. In order to achieve the objective, quality models from both aspects were reviewed along with LO concepts and quality characteristics that a LO must accomplish were determined in order to consider the evaluation characteristics. The IQMC method was used to construct the diagram for strategic (SD) $i^{*}$ sections for the final quality model. With the SD model created, it was established the traceability between the generated model criteria and the ISO/IEC 25010 matrix to keep a correlation between the $i^{*}$ notation characteristics and the standard ISO matrix mentioned before. The matrix obtained was enriched with conceptual characteristics and with the attributes that a LO should fulfill. For each quality property included in final model was asigned appropiate metrics for your evaluation. As a result, a model through LO evaluation with each one of the selected metrics was generated.
\end{abstract}

Keywords: quality model; i notation; learning object; IQMC; ISO/IEC 25010.

\section{Introducción}

Los Objetos de Aprendizaje (OA) son recursos digitales o unidades didácticas que permiten el apoyo del aprendizaje, los cuales son independientes, autónomos, reutilizables según su contexto, mantienen una estructura y son autocontenibles, así mismo son localizables gracias a sus metadatos (Maldonado, J., \& Astudillo, G. 2014). Sin embargo, cómo evaluar la calidad de este tipo de materiales educativos es un reto que aún sigue abierto.

\footnotetext{
1 Universidad de Cuenca, Cuenca, Ecuador ( \{vanessa.solis, jorge.maldonado\} @ucuenca.edu.ec ).

2 Universidad del Azuay, Cuenca, Ecuador ( jpcarvallo@uazuay.edu.ec ).
} 
La calidad de un OA puede ser evaluada desde diferentes perspectivas. Desde el punto de vista de su producción o desde el punto de vista como producto (Lavín, Del Solar, Fischer, \& Ibarra, 2002). Los modelos de calidad son importantes puesto que permiten medir la integración de distintos tipos de componentes. Para el caso de los OA elementos tecnológicos y didácticos. Grandes son los retos a la hora de evaluar el contenido y material creados que forman parte de MOOCs (Yamba y Luján, 2017) o cursos virtuales (Cedillo y otros, 2018) orientados a diferentes públicos.

Para medir la efectividad de los OA se han definido modelos de calidad orientados a evaluar la parte tecnológica. Por ejemplo, LORI (2003), APROA (2004), COdA (2012) o HEODAR (2005) entre otros son ejemplos de modelos desarrollados para evaluar la calidad de los OA. Sin embargo, algunos de estos modelos han dejado de lado componentes como el pedagógico o inclusive el didáctico. Es por esta razón que no se encuentran modelos que puede generalizar un artefacto con el propósito de poder medir la calidad de los OA de forma integral.

El objetivo de este trabajo es definir un nuevo modelo de evaluación de calidad de OA basándose en los modelos ya planteados, pero agregando como propuesta una matriz con atributos y métricas de evaluación que no han sido consideradas previamente. El nuevo modelo responde a la necesidad que los modelos actuales se enfocan a aspectos distintos (p.e. tecnológico, pedagógico) sin que hayan sido integrados en un modelo que recoja los aspectos tecnológicos y didácticos en un solo artefacto bajo los nuevos cambios tecnológicos que han surgido con el paso del tiempo.

Para lograrlo, se han considerado de forma prioritaria los ámbitos tecnológicos y didácticos ya que son los principales pilares y están directamente vinculados con la estructura de un OA, es decir en estos parámetros son relevantes para la construcción del material.

El artículo tiene la siguiente estructura. La Sección 2 presenta la metodología con la cual fue construido el nuevo modelo de calidad, la Sección 3 indica los resultados obtenidos con la matriz de calidad generada y la Sección 4 presenta las conclusiones a las que se llegó con la obtención de los resultados al evaluar un OA de ejemplo.

En este artículo inicialmente se describe el método utilizado para la generación del modelo de calidad, luego se hace una revisión de la bibliografía para extraer las características de varios modelos ya desarrollados, y realizar un análisis e identificar los actores que están directamente vinculados con el OA para a continuación determinar todas las características y atributos de los diferentes modelos y conceptos extraídos en la matriz resultante.

Al final hay que agregar una línea que indique cual es el aporte de este artículo para los investigadores.

\section{Metodología}

Para la elaboración del modelo de calidad para la evaluación de un OA se utilizó el método Individual Quality Model Construction (IQMC) (Carvallo, Franch, 2003). Este método permite construir de manera iterativa e incremental modelos de calidad para distintos dominios de software, partiendo de un catálogo inicial, en este caso el propuesto en el estandar ISO/IEC 25010. La determinación de las diferentes características, subcaracterísticas, atributos y métricas, que conforman el nuevo modelo de calida para los $\mathrm{OA}$, incluye las propiedades de calidad identificadas en la literatura, así como también en los nuevos parámetros detectados a base de cada uno de los actores vinculados en el modelo Diagrama de Dependencias(SD). El metodo IQMC incluye los siguientes pasos:

- Determinación de características de calidad de alto nivel.

- Determinación de subcaracterísticas de calidad.

- Determinación de atributos derivados.

- Determinación de atributos básicos.

- Establecimiento de relaciones entre factores de calidad. 
- Determinación de métricas para los atributos.

A continuación, se describe el proceso de construcción del modelo de contexto para la evaluación de calidad de un OA, considerando las etapas propuestas en el método IQMC, así como las diferentes características del estándar ISO/IEC 25010 (Paulsson \& Naeve, 2007) retroalimentado con los diferentes criterios, que han sido identificados en el modelo de contexto definido para los OA. Todas las características y atributos son enmarcados en los aspectos tecnológicos y didácticos.

La revisión bibliográfica abarcó la búsqueda de definiciones y conceptos de un OA. Posteriormente se investigaron trabajos propuestos por los autores vinculados con las definiciones de $O A$, verificando si estos desarrollaron métodos o modelos de evaluación para los mismos. De esta manera se fueron abstrayendo características o atributos que los OA deben cumplir en los ámbitos tecnológico y didáctico.

En la Figura 1 se indican los pasos que bajo IQMC se han seguido para obtener la matriz resultante de calidad para OA, cada uno de estos pasos se detallan en esta sección.

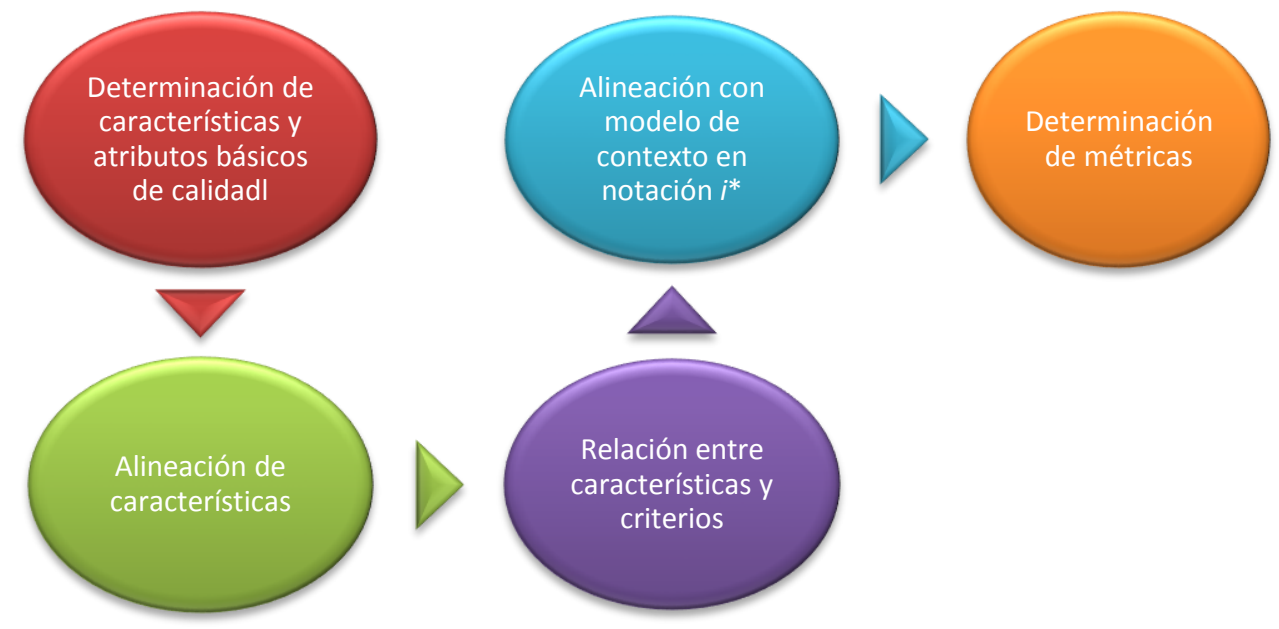

Figura 1. Pasos realizados para la generación de la matriz de calidad de OA

\subsection{Determinación de características y atributos básicos de calidad}

Una vez realizada la revisión bibliográfica de los diferentes trabajos desarrollados sobre OA y modelos de evaluación de los mismos, se extrajeron las principales características de un $\mathrm{OA}$, estas son: interoperabilidad, granularidad, publicable y reutilizable, el OA también debe ser considerado con las definiciones presentadas en la Tabla 1.

Para incrementar las características y subcaracterísticas del nuevo modelo de calidad se consideraron las particularidades de cada uno de los modelos de metadatos, resumidos en la Tabla 2. Un metadato al ser información acerca del recurso y en este caso de un OA, permite obtener las características de los archivos que contienen los OA de manera digital, por esta razón estas características permitieron complementar el modelo de evaluación para su posterior inclusión o subdivisión de otros atributos. Adicionalmente cada uno de los modelos de metadatos permitieron incorporar directamente métricas, ya que, son parámetros que al ser básicos permiten la evaluación directa del OA.

De manera similar, luego de la investigación de los modelos de metadatos enfocados al ámbito educativo, se extrajeron las características relevantes (ver Tabla 3) para posteriormente considerarlas a estas dentro del nuevo modelo, según las características generales de la ISO/IEC25010. 
Tabla 1. Resumen de definiciones de un OA

\begin{tabular}{|c|c|c|c|c|c|c|c|c|}
\hline Definición & 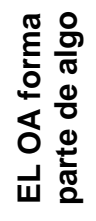 & 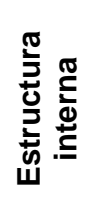 & 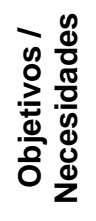 & 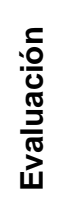 & 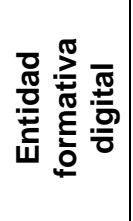 & 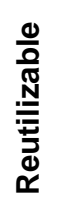 & 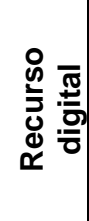 & 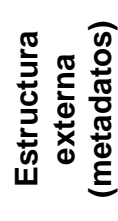 \\
\hline $\begin{array}{l}\text { Real Academia Española (Española, } \\
\text { 2016) }\end{array}$ & $\mathrm{x}$ & & & & & & & \\
\hline David Merril (Española, 2016) & & $\mathrm{X}$ & & & & & & \\
\hline $\begin{array}{l}\text { Wayne Hodgins (Merrill, Li, \& Jones, } \\
\text { 1991) }\end{array}$ & $\mathrm{x}$ & $\mathrm{x}$ & & & & & & \\
\hline James L'allier (Hodgins H. W., 2000) & $\mathrm{X}$ & & $\mathrm{X}$ & $\mathrm{X}$ & & & & \\
\hline Ramírez (Ramírez, 2007) & & & $\mathrm{X}$ & & $\mathrm{X}$ & & & \\
\hline Cisco System (Barritt \& Lewis, 1999) & & & & & & $\mathrm{X}$ & & \\
\hline Barrit \& Lewis (Barritt \& Lewis, 1999) & & & & & & $\mathrm{X}$ & & \\
\hline $\begin{array}{l}\text { IEEE (IEEE Learning Technology } \\
\text { Standards Committee, 2002) }\end{array}$ & $\mathrm{x}$ & $\mathrm{x}$ & & & & $\mathrm{X}$ & $\mathrm{X}$ & \\
\hline Wiley (Wiley, 2000) & & & & & & $\mathrm{X}$ & $\mathrm{X}$ & \\
\hline Chan Núñez (Chan Núñez, 2002) & $\mathrm{X}$ & & $\mathrm{X}$ & & & & $\mathrm{X}$ & \\
\hline Polsani (Polsani, 2003) & $\mathrm{X}$ & & & & & $\mathrm{X}$ & & \\
\hline Mc Greal (McGreal, 2004) & $\mathrm{X}$ & & & & & $\mathrm{X}$ & $\mathrm{X}$ & \\
\hline Zapata Ros (Zapata Ros, 2005) & & & $\mathrm{X}$ & & & $\mathrm{X}$ & $\mathrm{X}$ & \\
\hline García Aretio (García Aretio, 2005) & $\mathrm{X}$ & & $\mathrm{X}$ & & & $\mathrm{X}$ & $\mathrm{X}$ & \\
\hline $\begin{array}{l}\text { Ministerio de Educación Nacional de } \\
\text { Colombia (Ministerio de Educación } \\
\text { Nacional de Colombia, 2006) }\end{array}$ & & & $\mathrm{x}$ & & & $\mathrm{x}$ & $\mathrm{x}$ & $\mathrm{x}$ \\
\hline Chiappe (Chiappe, 2007) & $\mathrm{X}$ & & $\mathrm{X}$ & & & $\mathrm{X}$ & & $\mathrm{X}$ \\
\hline $\begin{array}{l}\text { Martínez Naharro (Martínez Naharro, } \\
\text { 2007) }\end{array}$ & $\mathrm{x}$ & & & & & $\mathrm{x}$ & $\mathrm{x}$ & \\
\hline $\begin{array}{l}\text { González-Gamone (Gonzalez- } \\
\text { Barnone, 2008) }\end{array}$ & $\mathrm{x}$ & & & & & $\mathrm{x}$ & & \\
\hline $\begin{array}{l}\text { Sicilia \& Sánchez (Sicilia \& Sánchez, } \\
\text { 2009) }\end{array}$ & $\mathrm{x}$ & & & & & $\mathrm{x}$ & $\mathrm{x}$ & $X$ \\
\hline $\begin{array}{l}\text { Astudillo, Sanz \& Wilging (Astudillo, } \\
\text { Sanz, \& Willging, 2011) }\end{array}$ & $\mathrm{x}$ & & $\mathrm{x}$ & & & $\mathrm{x}$ & $\mathrm{x}$ & $\mathrm{x}$ \\
\hline Sanz (Sanz, 2014) & & & $\mathrm{X}$ & $\mathrm{X}$ & & & $\mathrm{X}$ & $\mathrm{X}$ \\
\hline
\end{tabular}

Tabla 2. Resumen de características de los modelos de metadatos

\begin{tabular}{|c|c|c|c|c|c|c|c|}
\hline \multirow[b]{2}{*}{ Modelo } & \multicolumn{7}{|c|}{ Características } \\
\hline & 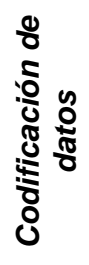 & 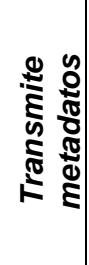 & 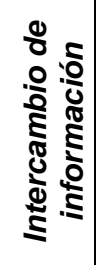 & 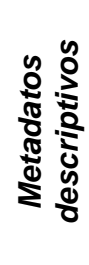 & $\frac{8}{3}$ & 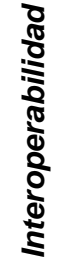 & 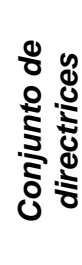 \\
\hline Dublin Core Metadata Iniciative-DC (Sanz, 2014) & $\mathrm{X}$ & $\mathrm{X}$ & $\mathrm{X}$ & $\mathrm{X}$ & $\mathrm{X}$ & $\mathrm{X}$ & \\
\hline $\begin{array}{l}\text { Metadata Encoding and Transmission Standard - METS } \\
\text { (METS, 2016) }\end{array}$ & $\bar{x}$ & $x$ & $x$ & $X$ & $\mathrm{X}$ & & \\
\hline $\begin{array}{l}\text { Metadata Object Description Schema - MODS (MODS, } \\
\text { 2016) }\end{array}$ & $\mathrm{X}$ & $\mathrm{X}$ & $\mathrm{X}$ & $X$ & & & \\
\hline Encoded Archival Description - EAD (EAD, 2016) & $\mathrm{X}$ & & $\mathrm{X}$ & & & & \\
\hline Text Encoding Initiative - TEI (TEI, 2016) & $\mathrm{X}$ & & $\mathrm{X}$ & & & & $\mathrm{X}$ \\
\hline $\begin{array}{l}\text { Metadata Resources for Digital Libraries - IFLA (IFLA, } \\
\text { 2015) }\end{array}$ & $\mathrm{X}$ & & $\mathrm{X}$ & & & & $\mathrm{X}$ \\
\hline
\end{tabular}


Tabla 3. Características de metadatos enfocados al ámbito educativo

\begin{tabular}{|c|c|c|c|c|c|c|}
\hline \multirow{2}{*}{$\begin{array}{l}\text { Modelos de metadatos } \\
\text { con enfoque educativo }\end{array}$} & \multicolumn{6}{|c|}{ Características } \\
\hline & 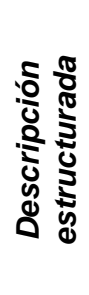 & 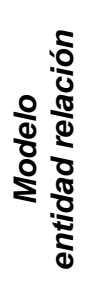 & 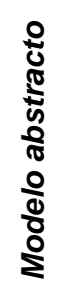 & 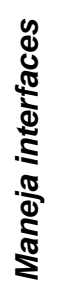 & 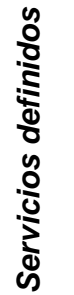 & 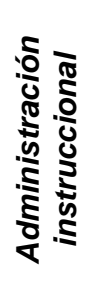 \\
\hline $\begin{array}{l}\text { Learning Object Metadata - LOM (IEEE, } \\
\text { 2016) }\end{array}$ & $x$ & & & & & \\
\hline $\begin{array}{l}\text { LOM-ES (Gobierno de España, 2010) } \\
\text { (Ministerio de Educación, 2010) }\end{array}$ & $x$ & & & & & \\
\hline Metadata Learning Resource - MLR [29] & & $\mathrm{X}$ & $x$ & & & \\
\hline $\begin{array}{l}\text { Instructional Management Systems - IMS ( } \\
\text { IMS Global Learning Consortium Inc., 2003) }\end{array}$ & & & & $\mathrm{X}$ & $\mathrm{X}$ & $\mathrm{X}$ \\
\hline $\begin{array}{l}\text { The Gateway to Educational Materials - } \\
\text { GEM (GEM, 2013) (DCMl, 2016) }\end{array}$ & $\mathrm{X}$ & & & & $\mathrm{x}$ & \\
\hline
\end{tabular}

\subsection{Alineación de características}

Se realizó un estudio comparativo, seleccionando todas las características relevantes de un $\mathrm{OA}$, las mismas que se recopilaron de la bibliografía contrastando con los diferentes modelos para evaluar la calidad en los OA. Este análisis permitió tener una visión clara de las diferentes características y subcaracterísticas que se incluirán y fortalecerán en la matriz ISO/IEC 25010, consideradas para la construcción del nuevo modelo de calidad para la evaluación de un OA.

Es así que, cada una de las particularidades y precisiones de los modelos, métodos, cuestionarios y herramientas detectadas en la teoría, al presentar alguna similitud tanto en nombres o definiciones, se consideró de forma única. En la Tabla 4 se presenta un fragmento del cruce de criterios, donde en la primera columna se presenta el listado de los diferentes modelos/herramienta revisada, en la segunda columna el nombre original de cada uno y finalmente como se determinó considerar a la característica para la construcción del modelo.

\subsection{Alineación con la notación $i^{\star}$}

La notación $i^{\star}$ se la utiliza para conceptualizar las diferentes dimensiones que comprenden las preguntas ¿qué?, ¿cómo?, ¿por qué?, es así que ha sido aplicado en diferentes áreas y gracias a la facilidad del manejo de las variantes ha logrado ser de gran utilidad (Wiki, 2015). Respondiendo a cada una de las preguntas se identificaron los actores vinculados con la creación, manipulación y utilización de un OA. En esta actividad se consideró el uso de la notación $i^{\star}$ para descomponer todo el entorno en el que se utiliza un OA. Es así que se identificaron 7 actores con actividades de elaborar/crear, utilizar, evaluar, comprender, publicar, contener y ejecutar un OA. Bajo estas premisas se describen las definiciones de los actores:

- Autor. quien realiza o desarrolla el OA. Un Docente también puede ser Autor.

- Docente: es el experto del tema que va a tratar el OA, quien genera el conocimiento para transmitirse en el OA. Un Docente también puede ser Autor. 
- Especialista en plataformas educativas: persona experta o especialista en plataformas educativas, es quien se encarga de los detalles de construcción en el ámbito técnico.

- Estudiantes: público para el que está enfocado el OA.

- Evaluador: experto que verificará el cumplimiento de los parámetros necesarios para que el OA tenga lo requerido para una buena ejecución. Cabe indicar que el evaluador también puede ser un Docente.

- Herramienta: consiste en cada uno de los instrumentos como programas, sistemas, plataformas que permiten generar, modificar, publicar un OA.

- Equipo: cada uno de los equipos que permite se pueda montar en él, algún tipo de software para utilizar el OA.

Tabla 4. Alineación de características

\begin{tabular}{|l|l|l|}
\hline \multicolumn{1}{|c|}{ Modelo/herramienta origen } & \multicolumn{1}{|c|}{ Nombre original } & \multicolumn{1}{c|}{ Criterio } \\
\hline $\begin{array}{l}\text { LORI (Nesbit, Belfer, \& Vargo, 2002) } \\
\text { COdA (Romero, Cesteros, \& de Armas Ranero, } \\
\text { 2012) }\end{array}$ & $\begin{array}{l}\text { Accesibilidad } \\
\text { Accesibilidad }\end{array}$ & Accesibilidad \\
\hline $\begin{array}{l}\text { Habilidades Metacognitivas (Menéndez, Castellanos, } \\
\text { Vidal, \& Segura, 2012) }\end{array}$ & $\begin{array}{l}\text { Adecuación a los } \\
\text { destinatarios }\end{array}$ & $\begin{array}{l}\text { Adecuación a los } \\
\text { destinatarios }\end{array}$ \\
\hline Paulsson y Naeve (Paulsson \& Naeve, 2007) & $\begin{array}{l}\text { Ampliación de los } \\
\text { estándares }\end{array}$ & $\begin{array}{l}\text { Ampliación de los } \\
\text { estándares }\end{array}$ \\
\hline $\begin{array}{l}\text { LORI } \\
\text { COdA } \\
\text { CUSEOA (Romero, Cesteros, \& de Armas Ranero, } \\
\text { 2012) } \\
\text { Paulsson y Naeve }\end{array}$ & $\begin{array}{l}\text { Calidad del contenido } \\
\text { Calidad de los } \\
\text { contenidos } \\
\text { Calidad del contenido } \\
\text { Definición ajustada }\end{array}$ & $\begin{array}{l}\text { Calidad del } \\
\text { contenido }\end{array}$ \\
\hline $\begin{array}{l}\text { CUSEOA } \\
\text { HEODAR (Morales \& et.al., 2005) } \\
\text { Paulsson y Naeve }\end{array}$ & $\begin{array}{l}\text { Diseño de } \\
\text { navegación }\end{array}$ & $\begin{array}{l}\text { Modelos } \\
\text { arquitectónicos }\end{array}$ \\
\hline
\end{tabular}

Toda esta identificación constituye la base para la construcción del modelo de calidad desde los diferentes ámbitos y actores que utilizan el OA (Franch \& Carvallo, 2003). Una vez identificados los actores, se modelaron cada una de las dependencias estratégicas (SD), donde constan objetivos (goals), objetivos blandos (quality) y recursos (resource) que permiten evaluar un OA. Un pequeño extracto se ha modelado como ejemplo en la Figura 1 y una versión tabular se presenta más ampliado en la Tabla 5, donde se puede evidenciar la relación de los actores con sus respectivos criterios con respecto al OA.

\subsection{Relación entre características y criterios}

A base de las 8 características que presenta la ISO/IEC 25010 (ISO 25000, 2015): adecuación funcional, eficiencia del desempeño, compatibilidad, usabilidad, fiabilidad, seguridad, mantenibilidad y portabilidad, se realizó la descomposición jerárquica los criterios establecidos en la Tabla 5 (se presenta un extracto de esta descomposición). Para nutrir la matriz resultante se consideraron cada uno de los aspectos tanto de forma tecnológica como didáctica extraídos de la literatura.

\subsection{Determinación de métricas}

En la matriz generada se asignó la métrica "True" (para casos en los que el OA cumpla con la característica. Ej. Dispone bibliografía para reforzar conocimientos) o False (para el caso que no cumpla). Cuando se trata de un porcentaje se lo presenta en forma decimal para poder contabilizarlo. 
Tabla 5 Dependencias estratégicas para la evaluación de un OA

\begin{tabular}{|c|c|c|c|}
\hline Actor & Criterio & $\begin{array}{l}\text { Tipo de } \\
\text { objetivo }\end{array}$ & Actor 2 \\
\hline \multirow{9}{*}{ 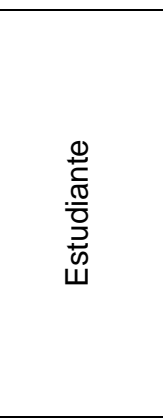 } & Accesibilidad & Quality & \multirow{3}{*}{$\mathrm{OA}$} \\
\hline & Adaptabilidad & Quality & \\
\hline & $\begin{array}{l}\text { Adecuación a los destinatarios } \\
\text { proporcionada }\end{array}$ & Quality & \\
\hline & Conexión a redes sociales proporcionada & Goal & \multirow{46}{*}{$\mathrm{OA}$} \\
\hline & Contenido comprensible & Quality & \\
\hline & Elementos de guía proporcionados & Goal & \\
\hline & Multimedia inteligible & Quality & \\
\hline & Traducciones realizadas & Goal & \\
\hline & Métodos abreviados utilizados & Goal & \\
\hline \multirow{6}{*}{ 京 } & Recursos de audiovisuales gestionados & Goal & \\
\hline & $\begin{array}{l}\text { Ayudas y menús contextuales } \\
\text { proporcionados }\end{array}$ & Goal & \\
\hline & Backups requeridos & Goal & \\
\hline & Errores gestionados & Goal & \\
\hline & Interfaces de presentación gestionadas & Goal & \\
\hline & Trabajo colaborativo gestionado & Goal & \\
\hline 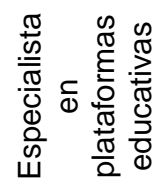 & Interoperabilidad & Quality & \\
\hline \multirow{5}{*}{ 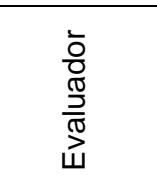 } & Aprobación proporcionada & Goal & \\
\hline & Evaluaciones gestionadas & Goal & \\
\hline & Apego al estándar SCORM & Resource & \\
\hline & Tiempo en el mercado proporcionado & Goal & \\
\hline & Utilización de estándares & Quality & \\
\hline \multirow{9}{*}{$\begin{array}{l}\stackrel{0}{\frac{1}{D}} \\
\stackrel{0}{0} \\
0\end{array}$} & $\begin{array}{l}\text { Actividades de reflexión, crítica e innovación } \\
\text { brindadas }\end{array}$ & Goal & \\
\hline & Aspectos didácticos & Quality & \\
\hline & Aspectos pedagógicos & Quality & \\
\hline & Contenido gestionado & Goal & \\
\hline & Contenidos evaluados & Goal & \\
\hline & Lenguaje correcto & Quality & \\
\hline & Lenguaje sencillo & Quality & \\
\hline & Participación activa & Quality & \\
\hline & Recursos y alianzas gestionados & Goal & \\
\hline \multirow{9}{*}{ 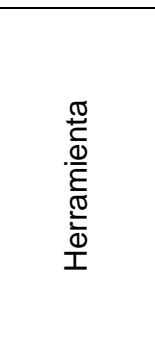 } & Accesos gestionados & Goal & \\
\hline & Ambientes gestionados & Goal & \\
\hline & Cuestionarios online proporcionados & Goal & \\
\hline & Duración OA requerido & Goal & \\
\hline & Encriptación gestionada & Goal & \\
\hline & Errores de usuario gestionados & Goal & \\
\hline & Sentencias ejecutadas & Goal & \\
\hline & Sistema Operativo utilizado & Goal & \\
\hline & Usuarios gestionados & Goal & \\
\hline \multirow{10}{*}{$\frac{\stackrel{\circ}{\circ}}{\frac{0}{5}}$} & Espacio en disco requerido & Goal & \\
\hline & Rendimiento requerido & Goal & \\
\hline & Tiempo de carga requerido & Goal & \\
\hline & Tiempo de ejecución realizado & Goal & \\
\hline & Tiempo de login requerido & Goal & \\
\hline & Uso CPU requerido & Goal & \\
\hline & Uso de red requerido & Goal & \\
\hline & Uso dispositivos $\mathrm{E} / \mathrm{S}$ requerido & Goal & \\
\hline & Uso GPU requerido & Goal & \\
\hline & Uso RAM requerido & Goal & \\
\hline
\end{tabular}


182

Es así que la matriz final contiene 249 métricas para la evaluación de calidad del OA. La Tabla 6 presenta un fragmento del modelo para evaluación de calidad con el extracto de las características y atributos correspondientes a la sección de Completitud de la implementación.

Tabla 6. Asignación de métricas

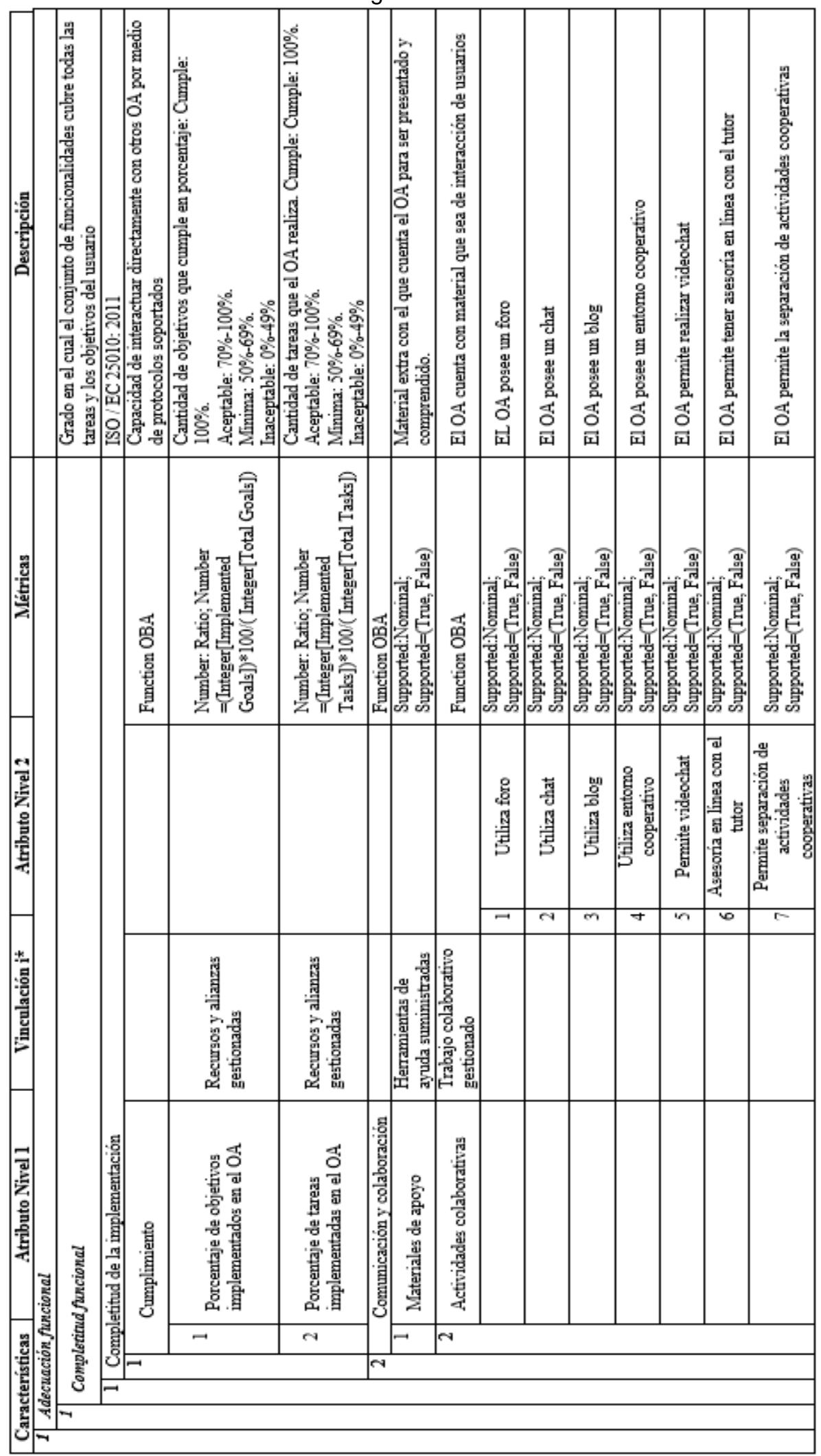

Enfoque UTE, V.9-N.2, Jun.2018, pp. $175-187$ 


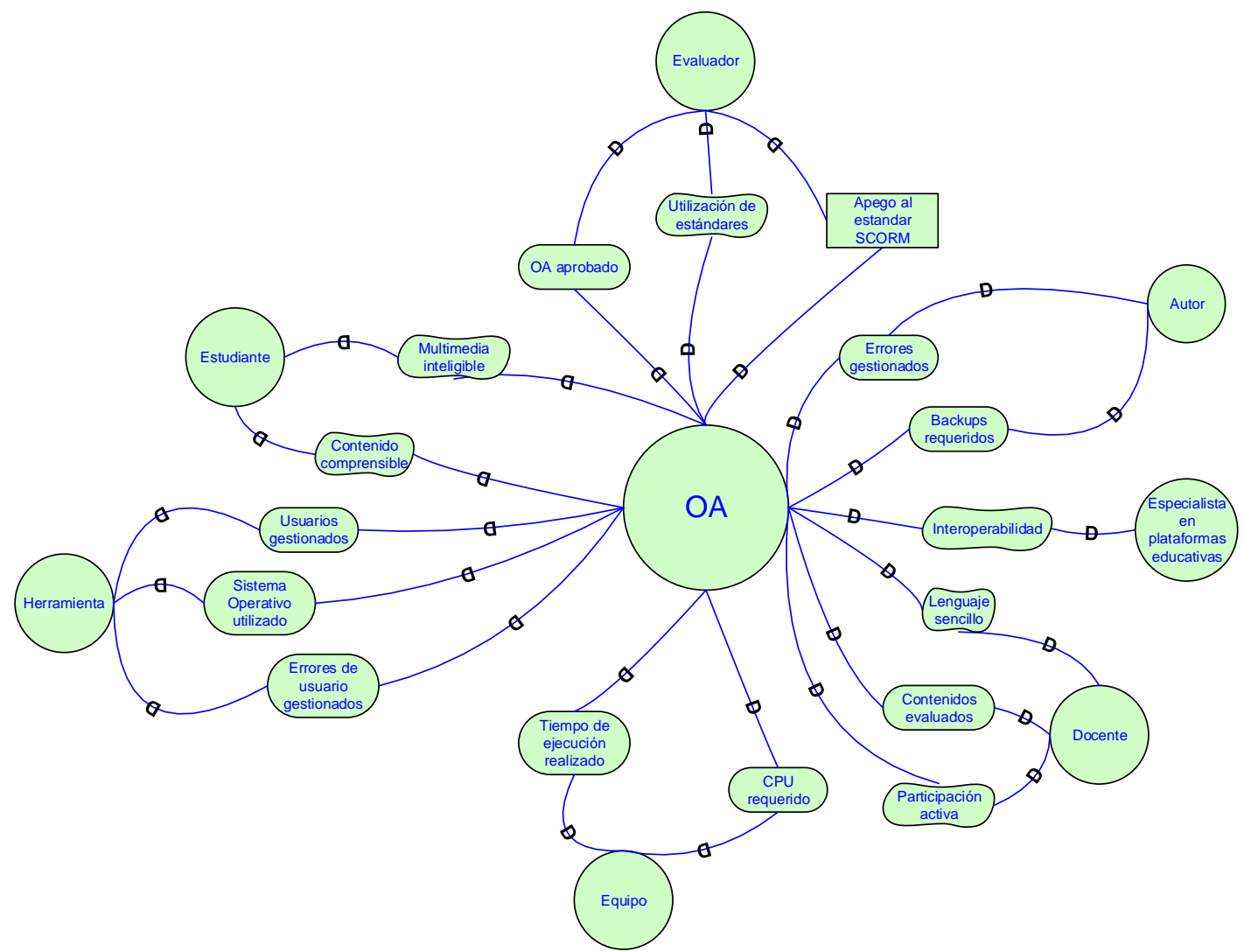

Figura 1. Identificación de actores con sus respectivos atributos a evaluarse

\section{Resultados}

Luego de construir la matriz de evaluación y asignar las métricas descritas, se consideró un $\mathrm{OA}$, el mismo que ha sido obtenido de un repositorio de OA (ROA) para la evaluación y efectos de demostración. En la Tabla 7 , se presenta un fragmento de la evaluación del OA, considerando las características, atributos y métricas establecidas.

Tabla 7. Calificación del OA

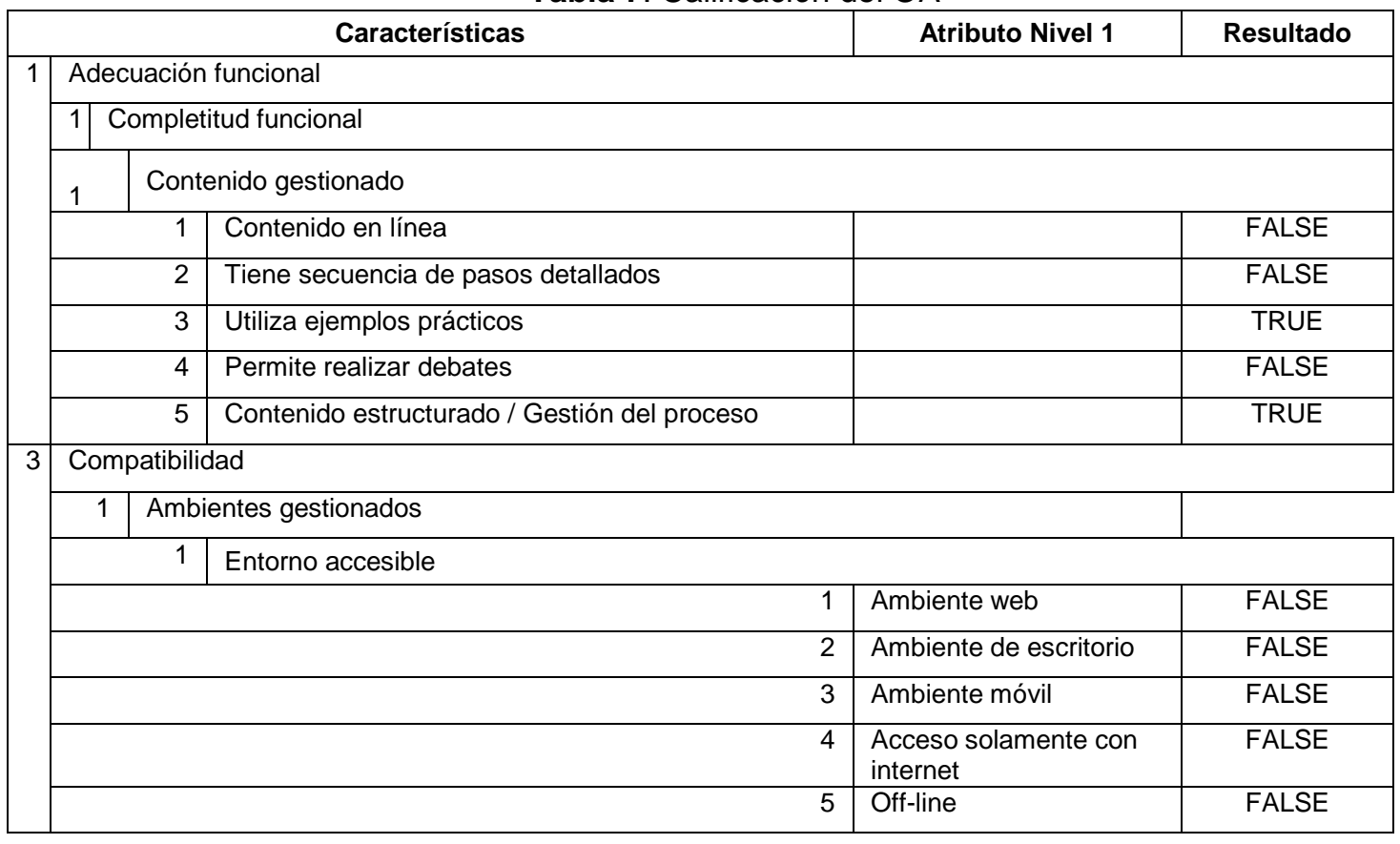


Bajo todos los criterios y parámetros establecidos en los ámbitos tecnológicos y didácticos, el OA obtiene cumple con 47 de los 249 criterios de evaluación completando así un 18,88\%, desglosado en la Tabla 8, según la categorización de la ISO/IEC 25010:

Tabla 8. Resultados de la evaluación

\begin{tabular}{|c|l|c|c|c|c|}
\hline No. & \multicolumn{1}{|c|}{ Característica } & TRUE & $\%$ & FALSE & $\%$ \\
\hline $\mathbf{1}$ & Completitud funcional & 11 & 19,30 & 46 & 80,70 \\
\hline $\mathbf{2}$ & Eficiencia de desempeño & 18 & 27,27 & 48 & 72,73 \\
\hline $\mathbf{3}$ & Compatibilidad & 2 & 16,67 & 10 & 83,33 \\
\hline $\mathbf{4}$ & Usabilidad & 11 & 17,46 & 52 & 82,54 \\
\hline $\mathbf{5}$ & Fiabilidad & 1 & 8,33 & 11 & 91,67 \\
\hline $\mathbf{6}$ & Seguridad & 0 & 0,00 & 11 & 100,00 \\
\hline $\mathbf{7}$ & Mantenibilidad & 2 & 15,38 & 11 & 84,62 \\
\hline $\mathbf{8}$ & Portabilidad & 2 & 13,33 & 13 & 86,67 \\
\hline \multicolumn{2}{r|}{ TOTAL } & 47 & 18,88 & 202 & 81,12 \\
\hline
\end{tabular}

\section{Conclusiones}

Actualmente los OA se están difundiendo de diferentes maneras, pero se debe considerar el hecho que los OA deben estar verificados para así garantizar su calidad. Este nuevo modelo de calidad permite tanto a técnicos como docentes tener una visión clara de cómo está el trabajo plasmado en un OA para la transmisión de conocimientos y el correcto funcionamiento del mismo, permitiendo así la mejora del material que se utilice para impartir conocimientos. Los parámetros de evaluación de OA enmarcados en calidad de modelos existentes, han sido considerados de los diferentes trabajos al respecto, donde se indican características básicas que debe tener un OA.

A base de los conceptos descritos por cada uno de los autores con relación a los OA, además de cada uno de los conceptos dentro de los modelos de aprendizaje tanto técnicos como educativos, se han extraído las características más relevantes tanto en el ámbito tecnológico como didáctico para la evaluación de OA. Así mismo se ha utilizado el método IQMC para desarrollar un modelo de dependencias estratégicas que vincula a todas las características recopiladas en la bibliografía. Finalmente se generó la trazabilidad entre cada uno de los actores del modelo mediante $i^{*}$ que presentaron sus Goals y Qualities dentro de las características y atributos en la matriz ISO 25010.

\section{Bibliografía}

APROA. (2004). EDU APROA Aprendiendo con objetos de aprendizaje. Obtenido de APROA: http://www.aproa.cl/

Astudillo, G., Sanz, C., \& Willging, P. (2011). Análisis del estado del arte de los objetos de aprendizaje. Revisión de su definición y sus posibilidades (Trabajo final). Recuperado el 2016, de http://sedici.unlp.edu.ar/ARG-UNLP-TPG-0000002954/12061.pdf

Barritt, C., \& Lewis, D. \&. (1999). Cisco Systems Reusable Information Object Strategy. Definition, Creation Overview, and Guidelines. Cisco Systems, Inc. Recuperado el 10 de Julio de 2016, de http://www.cisco.com/warp/public/779/ibs/solutions /learning/whitepapers/el_cis

Belloch, C. (2013). Diseño instruccional. Obtenido de http://www.uv.es/bellochc /pedagogia/EVA4.pdf

Cedillo Orellana, I., Beltrán, P., Rodríguez, P., Serrano, F., \& Bermeo, A. (2018). MOOCEP: Un método para construir cursos masivos para Adultos Mayores: Usando una creación MOOCEP. Enfoque UTE, 9(1), pp. 25 - 33. https://doi.org/10.29019 /enfoqueute.v9n1.242 
Chan Núñez, M. E. (2002). OBJETOS DE APRENDIZAJE: una herramienta para la innovación educativa. INNOVA, Objetos de aprendizaje. Experiencias de innovación educativa en los Centros de la Red Universitaria, 2, 3-11. Recuperado el 10 de Julio de 2016, de http://cvonline.uaeh.edu.mx/Cursos/ObjetosAprendizaje/PDF /STModulo01/lec_oa_htainnovacion.pdf

Chiappe, A. (2007). Acerca de lo pedagógico en los objetos de aprendizaje reflexiones conceptuales hacia la construcción de su estructura teórica. Estudios Pedagógicos, XXXV(1), 261-272. Recuperado el 20 de Julio de 2016, de http://www.redalyc.org/src/inicio/ArtPdfRed.jsp?iCve=173514138016

Carvallo, J.P., Franch X (2003). Using quality models in software package selection*. IEEE software.

DCMI. (2016). Dublin Core Metadata Innovation. Obtenido de The Metadata Community Supporting Innovation in Metadata Design, Implementation \& Best Practices: http://dublincore.org/

DCMI. (2016). Gateway to Educational Materials (GEM). Obtenido de GEM: http://dublincore.org/groups/education/GEM-Study.html

DCMI. (2016). Learning Resource Metadata Initiative. Obtenido de http://www.Irmi.net/

EAD. (26 de agosto de 2016). Encoded Archival Description. Obtenido de General information: https://www.loc.gov/ead/

Española, R. A. (10 de junio de 2016). Real Academia Española. Obtenido de Diccionario de la lengua española | Edición del Tricentenario: http://www.rae.es/

Franch, X., \& Carvallo, J. P. (2003). Using Quality Models in Software Package Selection. IEEE Software, http://computer.org/publications/dlib, 34-41.

García Aretio, L. (2005). Grupo de Apoyo a la Teleenseñanza. (Colaboraciones especiales Lorenzo García Aretio) Recuperado el 12 de julio de 2016, de http://www.um.es: http://www.um.es/atica/gat/gat2/tema-delmes /colaboraciones-especiales-garciaaretio/

GEM. (2013). The Gateway to Educational Materials. Obtenido de http://www.thegateway.org/

Gobierno de España, P. (2010). Análisis del Perfil de Aplicación LOM-ES. Recuperado el 2017, de http://www.edu.xunta.es: http://www.edu.xunta.es/contidos/ODEs/lex2 /analisis_UNE_71361_perfil_de_aplicacion_LOM-ESv1.0.pdf

Gonzalez-Barnone, V. \&.-R. (2008). Creating the first SCORM object. Computers \& Education(4), 1634-1647.

Hodgins, H. W. (2000). The future of learning objects, The instructional use of learning objects. David A. Recuperado el 10 de Julio de 2016, de http://reusability.org/read/chapters/hodgins.doc

IEEE. (2016). IEEE Standards Association. Obtenido de IEEE Learning Techonology Standards Committee (LTSC) Systems Interoperability in Education and Training: http://ltsc.ieee.org/wg12/

IEEE Learning Technology Standards Committee. (2002). Draft Standard for Learning Object Metadata. Recuperado el 10 de julio de 2016, de http://ltsc.ieee.org/wg12/files/LOM_1484_12_1_v1_Final_Draft.pdf

IEEE-LTSC. (2002). IEEE Standard for Learning Object Metadata. Obtenido de http://lsc.ieee.org/wg12/

IFLA. (9 de Julio de 2015). IFLA Standards. Obtenido de In support of quality library and information services worldwide: http://www.ifla.org/node/9337

IMS Global Learning Consortium Inc. (Julio de 2003). IMS Abstract Framework: White Paper. Obtenido de Versión 1.0: https://www.imsglobal.org/

ISO. (2009). ISO 15836:2009(en). Obtenido de http://www.iso.org/iso /catalogue_detail.htm?csnumber=52142: https://www.iso.org/obp/ui/\#!iso:std:52142:en

ISO 25000. (2015). ISO 25000. Obtenido de ISO/IEC 25010: http://www.iso25000.com /index.php/normas-iso-25000/iso-25010 
Lamarca, M. J. (2007). Hipertexto: El nuevo concepto de documento en la cultura de la imagen. Obtenido de www.terras.edu.ar: http://www.terras.edu.ar/aula/tecnicatura/3 /biblio/3Lamarca.pdf

Lavín, S., Del Solar, S., Fischer, M., \& \& Ibarra, J. C. (2002). La propuesta CIGA: gestión de calidad para instituciones educativas. 23.

Maldonado, J., \& Astudillo, G. (2014). Los Objetos de Aprendizaje: Un estado del arte en Iberoamérica. In VI Conferencia Iberoamericana sobre Tecnologías y Aprendizaje, Miami, Estados Unidos).

Martínez Naharro, S. B. (2007). Los objetos de aprendizaje como recurso de calidad para la docencia: criterios de validación de objetos en la Universidad Politécnica de Valencia. IV Simposio Pluridisciplinar sobre Diseño y Evaluación de Contenidos Educativos Reutilizables.

McGreal, R. (2004). Online Education using Learning Objects (2 ed.). (Routledge, Ed.) RouldledgeFalmer. doi:1134116799, 9781134116799

Menéndez, D. V., Castellanos, B. M., Vidal, C. C., \& Segura, A. (2012). Un modelo de calidad de Objetos de Aprendizaje basado en la semántica de sus metadatos. Obtenido de http://laclo.org/papers/index.php/laclo/article/download/51/46

Merrill, M. D., Li, Z., \& Jones, M. K. (1991). Second Generation Instructional Design (ID2) (Vol. 30). Utah State University. Recuperado el 8 de junio de 2016, de http://www.speakeasydesigns.com/SDSU/student/SAGE/compsprep/ID1_and_ID2.p df

METS. (9 de febrero de 2016). Metadata Encoding \& Transmission Standard. Obtenido de METS: Introducción y tutorial: http://www.loc.gov/standards/mets/

Ministerio de Educación Nacional de Colombia. (2006). ¿Qué es un Objeto de Aprendizaje? Obtenido de http://aprendeenlinea.udea.edu.co//ms/men /oac1.html

Ministerio de Educación, I. d. (8 de febrero de 2010). Perfil de aplicación LOM-ES V1.0. Recuperado el 27 de 04 de 2017, de http://www.edu.xunta.es/contidos/ODEs /lex2/herramientas.htm

MODS. (1 de febrero de 2016). Metadata Object Description Schema. Obtenido de MODS: Uses and Features: http://www.loc.gov/standards/mods/

Morales, E., García, F., Moreira, T., Rego, H., \& Berlanga, A. (2005). Valoración de la calidad de unidades de aprendizaje. Revista de Educación a Distancia, 1-13. Obtenido de Units of learning quality valuation: http://www.redalyc.org/html/547 /54709609/

Nesbit, J. C., Belfer, K., \& Vargo, J. (2002). A convergent participation model for evaluation of learning objects. Canadian Journal of Learning and Technology, 28(3), 105-120.

NISO. (2007). NISO Standards - National Information Standards Organization. Obtenido de NISO Standards: www.niso.org

Paulsson, F., \& Naeve, A. (2007). Establishing technical quality criteria for Learning Objects. 1431-1439.

Polsani, P. R. (2003). Use and Abuse of Reusable Learning Objects. Journal of Digital, 3(5). Obtenido de journals.tdl.org/jodi/article/viewArticle/89

Ramírez, M. S. (2007). Administración de objetos de aprendizaje en educación a distancia: experiencia de colaboración interinstitucional. En A. y. Lozano, Tecnología educativa en un modelo de educación a distancia centrado en la persona (Vol. 351, págs. 351373). Recuperado el 10 de julio de 2016, de http://www.ruv.itesm.mx/convenio /catedra/recursos/material/cl_01.pdf

Romero, E. D., Cesteros, A. F., \& de Armas Ranero, I. (2012). COdA, una herramienta experimentada para la evaluación de la calidad didáctica y tecnológica de los materiales didácticos digitales. RELADA-Revista Electrónica de ADA-Madrid, 6(4). Obtenido de http://polired.upm.es/index.php/relada/article/view/1925/1930

Sánchez, M. F., \& Castro, J. G. (2005). Calidad total: modelo EFQM de excelencia. F. C. Editorial. 
Sanz, C. (2014). Curso de Doctorado "Objetos de Aprendizaje”. Universidad Nacional de la Plata.

Sicilia, U. M.-A., \& Sánchez, A. S. (2009). Learning objects y learning designs: conceptos. Presentado en Diseño y Evaluación de contenidos y actividades educativas reutilizables, Information Engineering Research Unit.

TEl. (19 de Julio de 2016). TEl: Text Encoding Initiative. Obtenido de http://www.teic.org/index.xml

Wiki, i. (5 de agosto de 2015). $i^{\star}$ wiki (iStar 2.0 Core Language Istar). Obtenido de http://istar.rwth-aachen.de: http://istar.rwth-aachen.de

Wiley, D. A. (2000). Connecting learning objects to instructional design theory: a definition, a metaphor, and a taxonomy, the instructional use of learning objects. Recuperado el 10 de Julio de 2016, de http://reusability.org/read/chapters/wiley.doc

Yamba-Yugsi, M., \& Luján-Mora, S. (2017). Cursos MOOC: factores que disminuyen el abandono en los participantes. Enfoque UTE, 8(1), pp. 1 - 15. https://doi.org/10.29019 /enfoqueute.v8n1.124

Zapata Ros, M. (2005). Secuenciación de contenidos y objetos de aprendizaje. Revista de Educación a Distancia, Número monográfico II, 1-39. Recuperado el 10 de julio de 2016, de http://revistas.um.es/index.php/red/article/view/25221 